\title{
High pressure crystalline phase formation during nanoindentation: Amorphous versus crystalline silicon
}

\author{
S. Ruffell, ${ }^{\text {a) }}$ J. E. Bradby, and J. S. Williams \\ Department of Electronic Materials Engineering, Research School of Physical Sciences and Engineering, \\ Australian National University, Canberra 0200, Australia
}

(Received 18 May 2006; accepted 5 July 2006; published online 1 September 2006)

\begin{abstract}
Phase transformations induced by indentation at different unloading rates have been studied in crystalline and amorphous silicon via Raman microspectroscopy and transmission electron microscopy. Unloading was performed at a "slow" rate of $\sim 0.9 \mathrm{mN} / \mathrm{s}$ which is known to create volumes of high pressure phases ( $\mathrm{Si}$-III and Si-XII) in crystalline silicon as well as "rapid" unloading $(\sim 1000 \mathrm{mN} / \mathrm{s})$, where amorphous phases are expected. Stark differences between the resulting structures are observed depending on whether the starting material is amorphous or crystalline silicon. Interestingly, amorphous silicon transforms to high pressure phases much more readily than crystalline silicon even after rapid unloading. (C) 2006 American Institute of Physics. [DOI: 10.1063/1.2339039]
\end{abstract}

Silicon is a technologically important material having a wide range of applications. Consequently, in the last few decades, there has been great interest in its mechanical properties $^{1-10}$ most particularly the phase transformations that occur during nanoindentation. Diamond cubic Si-I is known to transform to a metallic Si-II phase at a pressure of $\sim 11 \mathrm{GPa}^{9,10}$ and further phase transforms (depending on unloading conditions) to amorphous silicon $(a-\mathrm{Si})$ and/or the high pressure crystalline phases $\mathrm{Si}$-III and $\mathrm{Si}$-XII on unloading. 5,6,11 Some recent work on ion-implanted $a$-Si (Ref. 12) suggests that $a$-Si behaves in the same way provided it is in a relaxed state which is achieved by annealing at $450{ }^{\circ} \mathrm{C}$ following implantation. ${ }^{12,13}$ The phase transformations on unloading are still not fully understood and differences between crystalline silicon $(c-\mathrm{Si})$ and $a$-Si have not been studied in detail. The aim of this study is to further the understanding of the transformation mechanisms for both $c$-Si and $a$-Si by examining end phases following unloading at various rates.

Crystalline samples were cleaved from a Czochralski grown $\operatorname{Si}(100)$ wafer $p$ doped with boron to a resistivity of $8-12 \Omega \mathrm{cm}$. The $a$-Si samples were created by ion implantation into the same wafers with various energies and fluences of Si ions followed by an anneal at $450{ }^{\circ} \mathrm{C}$ in an argon ambient for $30 \mathrm{~min}$ leaving a $2 \mu \mathrm{m}$ thick surface layer of relaxed $a$-Si. Indentation was performed using an UltraMicro Indentation System-2000 (UMIS) and an $\sim 5 \mu \mathrm{m}$ spherical indenter. Unloading from $80 \mathrm{mN}$ in 40 increments (average rate of $\sim 0.9 \mathrm{mN} / \mathrm{s}$ ) was performed thus favoring a final structure consisting of Si-III and Si-XII only. ${ }^{3,5,14} \mathrm{Com}-$ plete unloading was also performed in a single increment (average unload rate of $\sim 1000 \mathrm{mN} / \mathrm{s}$ ) to investigate the extreme case where, for $c$-Si under these loading conditions, it has been observed that only $a$-Si is formed..$^{5}$ A Renishaw 2000 Raman microspectroscopy instrument using a $632.8 \mathrm{~nm}$ laser was used to characterize the structure of the phases after indentation. Cross-sectional transmission electron microscopy (XTEM) was performed using a Phillips

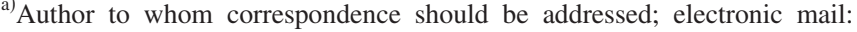
simon.ruffell@anu.edu.au
CM 300 transmission electron microscope from samples prepared using a focused ion beam milling and "pluck out" method. ${ }^{6,15,16}$ This allowed selected indents to be imaged and directly correlated with their specific load/unload curve and Raman spectrum.

Figure 1(a) shows an example of a complete load/unload curve for indentation in both relaxed $a$-Si and $c$-Si (unloading in 40 increments, $\sim 0.9 \mathrm{mN} / \mathrm{s}$ ). A pop-out event on each of the unloading portions of the curves is observed at $\sim 40 \mathrm{mN}$ indicative of a sudden increase in volume of material directly under the indenter tip and formation of $\mathrm{Si}$-III/ XII. The associated Raman spectra are shown in Fig. 1(b). Indents created in this manner in both $c$-Si and $a$-Si contain high pressure phases indicated by the extra peaks in the Raman spectra compared to those taken from pristine material. Indeed, XTEM images taken from these indents reveal indistinguishable indents that are completely filled with the high pressure phases as shown in the inset of Fig. 2(b).

For unloading from $80 \mathrm{mN}$ in one increment $(\sim 1000 \mathrm{mN} / \mathrm{s})$ only one data point (final point at $0 \mathrm{mN}$ ) was collected due to limitations of the UMIS instrument. It is, therefore, not possible to determine if a pop-out event occurs but it is unlikely. It has been shown that under these conditions for $c$-Si, unloading in less than four increments results in the formation of $a$-Si only. ${ }^{5,11}$ As expected, no evidence of high pressure phases is present in both the XTEM (Fig. 2) and Raman spectrum (not shown here) for the indent in $c$-Si with both showing evidence of $a$-Si only. A dramatic difference in behavior is observed for the indent in relaxed $a$-Si. The XTEM image shows a volume of the high pressure phases below the surface extending to the periphery of where the phase transformed Si-II would be located at the point of maximum load. These additional high pressure phases are also detected in the Raman spectrum. Although this XTEM image represents only a single indent, additional data ${ }^{17}$ indicate that substantial volumes of Si-III/XII are formed during rapid unloading of $a$-Si for more than 50 indents measured by Raman microspectroscopy. XTEM measurements have also made on indentations unloaded rapidly from different fractions of the maximum load (see next paragraph) revealing substantial volumes of Si-III/XII for all $a$-Si samples. ${ }^{17}$ 

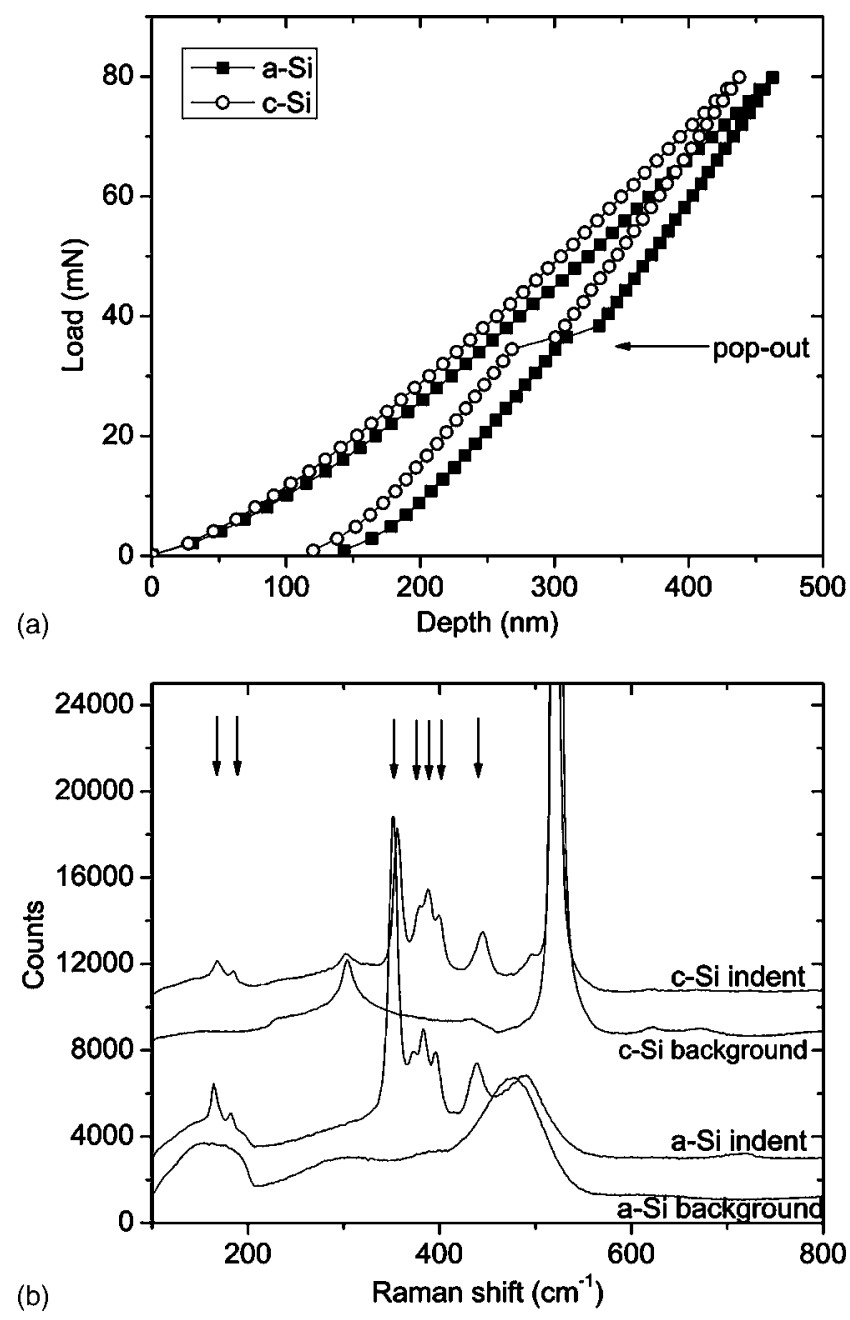

FIG. 1. (a) Load/unload curves from indentation in crystalline and relaxed amorphous silicon. As expected, slight differences in maximum penetration depth between the $c$-Si and $a-\mathrm{Si}$ are observed. Both loading and unloading were performed in 40 increments corresponding to an average rate of $\sim 0.9 \mathrm{mN} / \mathrm{s}$. For the rapid unloading from the maximum load no data during unloading are logged. The loading portions of these curves are the same as those shown here. (b) Raman spectra collected from the indents corresponding to the load/unload curves each showing the presence of high pressure phases. A spectrum from a pristine area of each of the samples is shown for reference. The additional peaks, marked by arrows $(167,182,353,378,387$, 399 , and $444 \mathrm{~cm}^{-1}$ ), originate from Si-III and $\mathrm{Si}-\mathrm{XII}$ phases.

In comparison, there is no evidence of these crystalline phases in $c$-Si following rapid unloading.

To further investigate the dependence of end phases on unloading conditions we began unloading rapidly from different points on the unload curves. ${ }^{17}$ Figure 3 shows the volume fraction of high pressure phases as determined from XTEM micrographs for rapid unloading from different fractions $(90 \%, 70 \%, 50 \%$, and $20 \%)$ of the maximum load. For points before the pop-out event $(\geqslant 50 \%$ of the maximum load), little or at most small volumes $(<6 \%)$ of high pressure phases are observed for the case of $c$-Si. In contrast, large volumes $(>50 \%)$ are observed for the case of $a$-Si.

The data presented here clearly show that the crystallization to Si-III/XII on unloading occurs much more readily when performing indentation in $a$-Si. This is a surprising result as one would expect crystallization to be favored for indentation in $c$-Si where a crystalline template exists and we discuss possible causes of this difference below. be directly compared. The dashed lines are to guide the eyes only.
Downloaded 14 Oct 2007 to 150.203 .178 .117 . Redistribution subject to AlP license or copyright, see http://apl.aip.org/apl/copyright.jsp

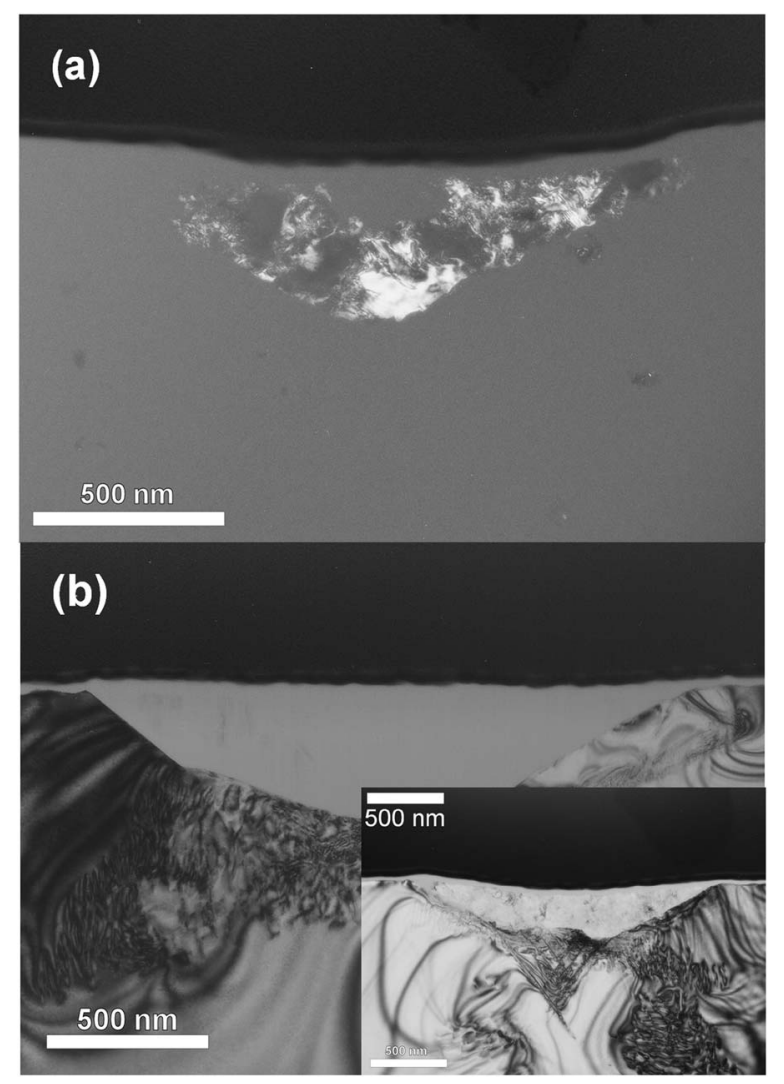

FIG. 2. Dark field XTEM images from indents in (a) amorphous silicon and (b) crystalline Si following a rapid unloading cycle. The indent in $a-\mathrm{Si}$ contains a volume of high pressure phase silicon (selected area diffraction pattern conforms this) as well as amorphous silicon at the surface. For the indent in $c$-Si the complete volume observed by TEM is $a$-Si. The associated Raman spectra (not shown) show evidence only of $a$-Si. The inset in (b) is taken from the indent in $c$-Si (that in $a$-Si is identical) with slow unloading. The whole indent is full of the high pressure phases. The associated Raman spectra are in Fig. 1 .

Firstly, the form of the metallic phase (crystalline $\beta$-Sn or high density $a-\mathrm{Si}$ ) is not known and may be different for indentation in the two different starting materials, as has been suggested in diamond anvil studies previously. ${ }^{18}$ For example, based on diamond anvil data the $\beta$-Sn phase is

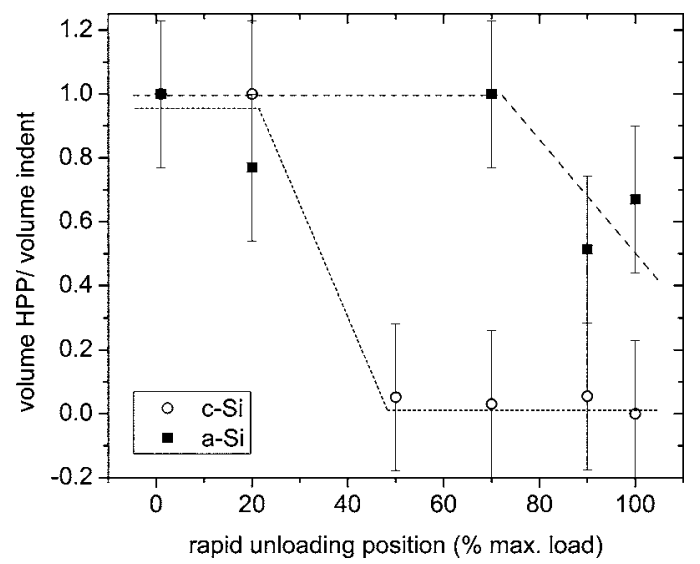

FIG. 3. Summary of XTEM data taken from indents in $a$-Si and $c$-Si. The plot shows the volume percentage of Si-III/XII as a function of the position of rapid unloading, as obtained from XTEM micrographs. The large error bars are estimated from measurements in which two XTEM images were collected for a particular unloading regime and could

be directly compared. The dashed lines are to guide the eyes only. 
expected for isostatic loading of $c$-Si, whereas it has been suggested that $a$-Si transforms to a (metallic) high density amorphous form on loading. If there are such differences in metallic phase in our indentation case, then, like the diamond anvil case, $c$-Si and $a$-Si may lead to different end phases on unloading. However, in the diamond anvil case $c$-Si transforms to high pressure crystalline phases whereas $a$-Si appears to transform back to low density $a$-Si on unloading. Our indentation case is contrary to this behavior. Therefore, it is unlikely that different inferred metallic phases can explain our results.

Another, possibly more important difference between the two systems is the interface formed by the metallic $\mathrm{Si}$ at maximum load with the surrounding matrix, i.e., $a$-Si or $c$-Si. It may be that the random nature of bonding arrangements in $a$-Si can provide an increased probability of nucleation of high pressure (crystalline) phases from the transforming metallic Si. In considering the kinetics of the phase transformation process, it has been previously suggested that the formation of high pressure phases is nucleation limited. ${ }^{5,14}$ The interface of the $a$-Si/Si-II may provide more suitable nucleation sites, thus increasing the probability for nucleation of Si-III/XII.

A further observation is that the phase transformation to $\mathrm{Si}$-III/XII during unloading in relaxed $a$-Si appears to initiate at the periphery of the volume of Si-II created at maximum load and extends towards the surface during unloading with the near surface volume appearing to transform back to $a$-Si. This suggests that the strain rate rather than the unload rate may dictate the phase transformation behavior, noting that at a constant unload rate, the strain relief rate increases as the indenter tip approaches the surface. Thus, $a$-Si formation may be favored at high strain rates. Other authors have also discussed transformation behavior in terms of strain rate rather than unloading rate. ${ }^{3,19-21}$ Indeed, Zarudi et al. ${ }^{19-21}$ suggested that differences in the stresses induced in the silicon during indentation (as a result of phase transformations) may cause differences in local strain relief rate and hence in subsequent phase transformation behavior.

In summary we have shown large differences in phase transformation behavior on unloading during indentation of $a-\mathrm{Si}$ and $c$-Si. For example, rapid unloading reveals that $a$-Si much more readily transforms to Si-III/XII with $\sim 50 \%$ of the indented volume of the indentation studied here containing high pressure phases compared to the $c$-Si case where the total indented volume contained only $a$-Si. There are a number of possible reasons for this. First, possible differences in the form the metallic silicon takes during indentation in $a$-Si compared with $c$-Si may lead to different end phases. However, a more plausible explanation would appear to be the interface formed between the metallic silicon and the surrounding matrix, whereby $a$-Si with variable bonding arrangements contributes more favorable nucleation sites for high pressure phase formation. Furthermore, the formation of $\mathrm{Si}$-III/XII appears to initiate from the periphery of the metallic $\mathrm{Si}$ volume but does not continue to the surface during rapid unloading, indicating that the rate of strain relief during unloading ultimately determines the volume of Si-III/XII once it has been nucleated.

The authors would like to thank P. Munroe and D. McGrouther from the Electron Microscopy Unit at the University of New South Wales for the use of their facilities and assistance in preparing the TEM samples. This work was funded by the Australian Research Council and WRiota Pty Ltd.

${ }^{1}$ D. R. Clarke, M. C. Kroll, P. D. Kirchner, R. F. Cook, and B. J. Hockey, Phys. Rev. Lett. 60, 2156 (1988).

${ }^{2}$ G. M. Pharr, W. C. Oliver, R. F. Cook, P. D. Kirchner, M. C. Kroll, T. R. Dinger, and D. R. Clarke, J. Mater. Res. 7, 961 (1992).

${ }^{3}$ A. Kailer, Y. G. Gogotsi, and K. G. Nickel, J. Appl. Phys. 81, 3057 (1997).

${ }^{4}$ Y. G. Gogotsi, V. Domnich, S. N. Dub, A. Kailer, and K. G. Nickel, J. Mater. Res. 15, 871 (2000).

${ }^{5}$ J. E. Bradby, J. S. Williams, J. Wong-Leung, M. V. Swain, and P. Munroe, J. Mater. Res. 16, 1500 (2001).

${ }^{6}$ J. E. Bradby, J. S. Williams, J. Wong-Leung, M. V. Swain, and P. Munroe, Appl. Phys. Lett. 77, 3749 (2000).

${ }^{7}$ J. Crain, G. J. Ackland, J. R. Maclean, R. O. Piltz, P. D. Hatton, and G. S. Pawley, Phys. Rev. B 50, 13043 (1994).

${ }^{8}$ R. O. Piltz, J. R. Maclean, S. J. Clark, G. J. Auckland, P. D. Hatton, and J. Crain, Phys. Rev. B 52, 4072 (1995).

${ }^{9}$ M. Imai, T. Mitamura, K. Yaoita, and K. Tsuji, High Press. Res. 15, 167 (1996).

${ }^{10}$ J. Z. Hu, L. D. Merkle, C. S. Menoni, and I. L. Spain, Phys. Rev. B 34, 4679 (1986).

${ }^{11}$ V. Domnich, Y. Gogotsi, and S. Dub, Appl. Phys. Lett. 76, 2214 (2000).

${ }^{12}$ B. Haberl, J. E. Bradby, S. Ruffell, J. S. Williams, and P. Munroe, J. Appl. Phys. 100, 013520 (2006).

${ }^{13}$ S. Roorda, W. C. Sinke, J. M. Poate, D. C. Jacobson, S. Dierker, B. S. Dennis, D. J. Eaglesham, F. Spaepen, and P. Fuoss, Phys. Rev. B 44, 3702 (1991).

${ }^{14}$ Jae-il Jang, M. J. Lance, Songqing Wen, Ting Y. Tsui, and G. M. Pharr, Acta Mater. 53, 1759 (2005).

${ }^{15}$ H. Saka, J. Vac. Sci. Technol. B 16, 2522 (1998).

${ }^{16}$ J. E. Bradby, Research School of Physical Sciences and Engineering, Australian National University, Canberra, 2002.

${ }^{17}$ S. Ruffell, J. E. Bradby, and J. S. Williams (unpublished).

${ }^{18}$ Sudip K. Deb, Martin Wilding, Maddury Somayazulu, and Paul F. McMillan, Nature (London) 414, 528 (2001).

${ }^{19}$ I. Zarudi, L. C. Zhang, and M. V. Swain, J. Mater. Res. 18, 758 (2003).

${ }^{20}$ I. Zarudi, L. C. Zhang, J. Zou, and T. Vodenitcharova, J. Mater. Res. 19, 332 (2004)

${ }^{21}$ I. Zarudi, L. C. Zhang, W. C. D. Cheong, and T. X. Yu, Acta Mater. 53, 4795 (2005). 